\title{
Vorwort der ASMET
}

Die Montanuniversität Leoben feiert 2015 ihr 175-jähriges Jubiläum. Der Start geht dabei auf das Gründungsjahr 1840 der Steiermärkisch-Ständischen Berg- und Hüttenmännischen Lehranstalt im Zentrum der steirischen Eisenproduktion in Vordernberg zurück. Sie übersiedelte in den folgenden Jahren nach Leoben und öffnete hier im Jahre 1849 ihre Pforten als einzige akademische Montanlehranstalt im deutschsprachigen Raum der österreichischungarischen Monarchie. Wie auch bei der Gründung von anderen Lehranstalten hat sich hier Erzherzog Johann dafür eingesetzt und bewirkt, dass damit eine zukunftsweisende Ausbildungseinrichtung geschaffen wurde. Aber eine Universität kann nicht alleine von ihrer Vergangenheit leben, das zeigt gerade die Montanuniversität Leoben. Die Montanuniversität hat sich stets den Blick für mittel- und langfristige Entwicklungen bewahrt und war erfolgreich bemüht, den Studierenden neben einer fundierten wissenschaftlichen Berufsvorbildung auch Unterstützung bei der persönlichen Entfaltung zu bieten. Die Zahl der ordentlichen Hörer an dieser Universität hat sich daher in den letzten 15 Jahren fast vervierfacht. Es ist gelungen, einerseits die klassischen montanistischen Studienrichtungen, getrieben durch die rasante technische Entwicklung, den modernen Gegebenheiten anzupassen, andererseits das Angebot an Studienrichtungen wesentlich zu erweitern. Die Absolventen sind in der Wirtschaft sehr begehrt, ihre Berufsaussichten sind überdurchschnittlich gut.

Insbesondere den hohen Anforderungen, die die Zukunft an Werkstoffe stellen wird, versucht die Montanuniversität Leoben bereits heute mit einer profunden Ausbildung gerecht zu werden. Die Forschungsergebnisse der Institute und der Kompetenzzentren bieten für die immer dringlicher werdenden weltweiten Probleme, wie z. B. Rohstoffmangel, Zerstörung der Umwelt, Lösungen an. In diesem Zusammenhang erweist es sich als Erfolgsfaktor, dass die in Leoben vertretenen Wissenschaftszweige in unmittelbarer Beziehung mit der betrieblichen Praxis mit Partnerfirmen stehen und so Forschungsergebnisse rasch nutzbringend Anwendung finden können.
Aus dem Selbstverständnis des Berg- und Hüttenstandes heraus war die Montanuniversität nie ein Elfenbeinturm. Sie hat immer die Kontakte mit der ihr zugeordneten Industrie gepflegt und dies - lange vor der Aufforderung der Öffentlichkeit an die Universitäten, sich dem Umfeld zu öffnen - aktiv vorgelebt. Auch heute zeichnet sich die Montanuniversität in einem besonderen Maße durch ihre Kooperationsfähigkeit und -willigkeit gegenüber Industrie und Wirtschaft aus. Für ihre Arbeit - ganz besonders auch für das beispielgebende Kooperationsverhältnis zwischen Universität und Industrie - gebührt der Montanuniversität Leoben Lob, Dank und Anerkennung.

Die Industrie und Gesellschaft in Europa, Österreich und damit auch die Montanuniversität stehen in ihrem Jubiläumsjahr vor großen Herausforderungen, die sie, wie in den letzten 175 Jahren, erfolgreich bewältigen wird - zum Wohle der Wirtschaft und der Menschen dieses Landes. Die Aufgaben, die vor uns liegen, sind herausfordernd. Österreich wird ihnen nur gewachsen sein, wenn es seinen hohen industriell-technischen Standard wahren und weiter ausbauen kann. Wir vertrauen dabei auch in Zukunft auf die bewährte Kreativität der Montanuniversität Leoben in Forschung und Lehre.

Namens der ASMET gratulieren wir der Montanuniversität Leoben sehr herzlich zum großen 175-Jahr-Jubiläum und wünschen ihr, ihren Professorinnen und Professoren und ihren Studentinnen und Studenten für die Zukunft alles Gute und weiterhin viel Erfolg mit einem herzlichen „Glückauf“!

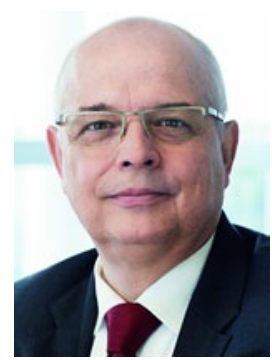

Franz Rotter



Bruno Hribernik 